\title{
Productive Cough, CTCAE
}

National Cancer Institute

\section{Source}

National Cancer Institute. Productive Cough, CTCAE. NCI Thesaurus. Code C143780.

A disorder characterized by expectorated secretions upon coughing. 